\title{
FLT3 Gene Mutation in Acute Myeloid Leukemia: Correlation with Hematological, Immunophenotypic, and Cytogenetic Characteristics
}

\author{
Sunitha Shankaralingappa ${ }^{1, \odot}$ Hemangi D. Joshi ${ }^{1}$ \\ ${ }^{1}$ Department of Oncopathology, The Gujarat Cancer Research \\ Institute, Ahmedabad, Gujarat, India \\ 2 Molecular and Cancer Biology, The Gujarat Cancer Research \\ Institute, Ahmedabad, Gujarat, India
}

\begin{abstract}
Jayendra B. Patel ${ }^{2} \quad$ Prabhudas Patel $^{2} \quad$ Jyoti Sawhney ${ }^{1}$
\end{abstract}
\author{
Address for correspondence Jyoti Sawhney, MD, DM, Department \\ of Oncopathology, The Gujarat Cancer Research Institute, \\ Ahmedabad 380016, Gujarat, India \\ (e-mail: jyoti.sawhney@gcriindia.org).
}

\begin{abstract}
Keywords

- acute myeloid leukemia

- FLT3-ITD

- FLT3-TKD

Introduction In acute myeloid leukemia (AML), FMS-like tyrosine kinase 3-internal tandem duplication (FLT3-ITD) is a common driver mutation associated with high tumor burden and poor prognosis. This mutation is common in normal karyotype AML and such patients have high leukocyte count. The presence of this mutation can be predicted by certain hematological and immunophenotypic characteristics in day-to-day practice.

Objective This study was undertaken to assess the strength of association between FLT3 gene mutation and hematological and immunophenotypic characteristics.

Materials and Methods Morphological, hematological, immunophenotypic, and cytogenetic characteristics of FLT3 mutations recorded in 424 patients of AML in adults and children between 2016 and 2019 in a tertiary care cancer center in Western India. Blasts were classified according to French-American-British method. Tumor burden was assessed by serum lactate dehydrogenase (LDH) levels, leucocyte count, and peripheral smear blast percentage.

Results Out of 424 cases, FLT3-ITD and FLT3-tyrosine kinase domain mutation were found in 72 and $25 \mathrm{AML}$ patients, respectively. Patients with FLT3 mutation had high tumor burden, characterized by high leukocyte count $(p<0.001)$, peripheral blood $(p=0.01)$ and bone marrow $(p=0.03)$ blast percentage, and high serum LDH (mean 777.8 vs. 586; $p=0.10$ ) compared with FLT3-negative patients. They also featured high platelet count $(p<0.001)$. Morphological, immunophenotypic, and cytogenetic characteristics also have been presented in the study.

Conclusion Observations of the study suggest the presence of definitive hematological and immunophenotypic characteristics along with raised serum LDH levels serve as surrogate markers and indicators of FLT3 mutation in AML patients.
\end{abstract}

published online Jun 18, 2021
DOI https://doi.org/

$10.1055 / \mathrm{s}-0041-1731091$ ISSN 2454-6798
(C) 2021. Spring Hope Cancer Foundation \& Young Oncologist Group of Asia.

This is an open access article published by Thieme under the terms of the Creative Commons Attribution-NonDerivative-NonCommercial-License, permitting copying and reproduction so long as the original work is given appropriate credit. Contents may not be used for commercial purposes, or adapted, remixed, transformed or built upon. (https://creativecommons.org/licenses/by-nc-nd/4.0/).

Thieme Medical and Scientific Publishers Pvt. Ltd. A-12, 2nd Floor, Sector 2, Noida-201301 UP, India 


\section{Introduction}

Acute myeloid leukemia (AML) is a malignancy of immature hematopoietic cells, characterized by clonal evolution and genetic heterogeneity. Several molecular aberrations have been proved to be important prognostic indicators. FMS-like tyrosine kinase 3-internal tandem duplication (FLT3-ITD) is a common driver mutation, seen with a frequency of 20 to $30 \%$ and significantly affecting the clinical outcome, especially in normal karyotype AML (NK-AML). Genetic testing for FLT3-ITD mutation at diagnosis is done at many centers as the standard of care to risk stratify patients and to guide therapeutic decisions in AML. ${ }^{1-3}$

FLT3 mutation also has an important role in pathogenesis of disease in AML. Both FLT3-ITD and FLT3-tyrosine kinase domain (FLT3-TKD) mutations lead to constitutive activation of FLT3 kinase and several downstream targets resulting in myeloproliferative phenotype and high tumor burden, a characteristic hematological and biochemical profile that can be identified during routine diagnostic workup. ${ }^{4-6}$ FLT3 in AML is also associated with characteristic immunophenotypic features. ${ }^{17,8}$

We retrospectively analyzed AML patients in this casecontrol study with the aim of evaluating the association of FLT3 mutation with hematological, biochemical, and immunophenotypic features. Genotypic analysis of this mutation in a cohort of West Indian population would help in understanding the pathogenesis of AML in mutant phenotype and thus guide to devise precise therapeutic intervention in such patients.

\section{Materials and Methods}

A retrospective cohort study was performed in the Department of Hematology, at a tertiary care cancer center in Western India with 424 ( 332 adults and 92 children) de novo cases of AML tested for FLT3-ITD and FLT3-TKD mutations during the period of 2016 to 2019. The diagnosis of AML was confirmed on morphology, cytochemistry, and flowcytometric immunophenotyping. Patients with acute promyelocytic leukemia and patients without any further molecular analysis were excluded from our study.

\section{Clinical, Hematological, and Biochemical Parameters}

Clinical features such as presence of hepatomegaly and splenomegaly were noted from radiology reports. Routine hematological investigations were performed by autoanalyzers Beckman LH-750 or Mindray BC- 6800 Plus, and serum LDH was measured by autoanalyzer Cobas 6000 . Hematological parameters including hemoglobin level $(\mathrm{g} / \mathrm{L})$, total leukocyte count $\left(\mathrm{x} 10^{3} / \mu \mathrm{L}\right)$, platelet count $\left(\mathrm{x} 10^{3} / \mu \mathrm{L}\right)$, peripheral as well as bone marrow blast percentages and serum $\mathrm{LDH}(\mathrm{U} / \mathrm{L})$ levels at the time of diagnosis were recorded and analyzed.

\section{Morphology and Cytochemistry}

Peripheral smear and bone marrow smears were stained with Leishman and Giemsa stain. Cytochemistry was performed on bone marrow smear by periodic acid Schiff (PAS) and Sudan Black (SB) stains to differentiate AML from acute lymphoblastic leukemia. Blasts in AML were either negative for PAS or showed diffuse cytoplasmic PAS positivity and were SB positive. Blasts were classified morphologically according to the French-American-British (FAB) classification from M1 to M7.

\section{Immunophenotyping}

Flow cytometry was performed on peripheral blood or bone marrow on eight color flow cytometer BD FACS Canto using Euroflow antibody panel by a standard procedure. Monoclonal antibodies used in the study were allophycocyanin (APC), allophycocyanin H7 (APC-H7), V450, V500, phycoerythrin Cy7 (PE-Cy7), peridinin chlorophyll-protein Cy5.5, PE and fluorescein isothiocyanate labeled CD33 and CD7, CD14 and cytoplasmic CD3, HLADR and surface CD3, CD45, CD117 and CD19, CD34 and CD5, CD13 and cytoplasmic CD79a (cCD79a) and cytoplasmic MPO (cMPO), CD15 and CD64. Blasts in AML showed expression for CMPO, CD34, CD117, HLADR, CD33, $C D 13, C 14$, and $C D 15$ in varying combinations depending on FAB category. Aberrant expression for CD7, CD19, CD5, and $\mathrm{CD} 3$ was detected.

\section{Cytogenetics}

Chromosomal abnormalities and cytogenetic aberrations were studied by conventional karyotyping and interphase fluorescent in-situ hybridization on bone marrow samples. Patients were divided into three subgroups in cases and controls: core-binding factor AML (CBF-AML) having $\mathrm{t}(8 ; 21)$ or inversion 16; NK-AML and high-risk AML having monosomy $7 / 7 \mathrm{q}$ deletion or monosomy $5 / 5 \mathrm{q}$ deletion or complex karyotype ( $\geq 3$ chromosomal abnormalities) or $\mathrm{t}(9 ; 22)$ or $11 \mathrm{q} 23$. Overall survival (OS), disease-free survival, and event-free survival were analyzed in different cytogenetic subgroups and compared between cases and controls.

\section{Molecular Testing-Analysis for FLT3-ITD and FLT3-TKD Mutation}

FLT3-ITD and TKD mutations were determined from genomic DNA using polymerase chain reaction (PCR)-based method, and subsequent restriction-digest with EcoRV endonuclease for the TKD reaction and size fractionation by capillary electrophoresis.

Peripheral blood samples of AML patients were collected in ethylenediaminetetraacetic acid vacutainer after informed consent. Genomic DNA was extracted using QIAamp DNA blood kit according to manufacturer's protocol (Qiagen, Hilden, Germany). Isolated DNA was amplified by semiquantitative PCR using primers GCAATTTAGGTATGAAAGCCAGC-3' (Forward 5') \& CTTTCAGCATTTTGACGGCAACC-3' (Reverse 5') for FLT3-ITD and CCGCCAGGAACGTGCTTG-3' (Forward 5') \& GCAGCCTCACATTGCCCC-3' (Reverse 5') for D835 mutation.

For FLT3-ITD mutation, a 200ng DNA was used as a template in PCR HimediaTaqmixture. Cycling conditions for the reaction were $94^{\circ} \mathrm{C}$ for 3 minutes, $94^{\circ} \mathrm{C}$ for 1 minute, $62^{\circ} \mathrm{C}$ for 1 minute, $72^{\circ} \mathrm{C}$ for 1 minute, and extension at $72^{\circ} \mathrm{C}$ for 10 minutes for total 40 cycles. PCR products were separated on $2 \%$ agarose gel stained with ethidium bromide. The gel 
was analyzed using Alpha Innotech system. FLT3 wild-type genotype was seen as band at 329bp, while an additional band above 329bp was observed in ITD mutation.

FLT3-TKD mutation was performed by PCR restriction fragment length polymorphism (PCR-RFLP) method. DNA of 300 ng was used as template in PCR Himedia Taq mixture. Cycling conditions for the reaction were $94^{\circ} \mathrm{C}$ for 3 minutes, $94^{\circ} \mathrm{C}$ for 1 minute, $65^{\circ} \mathrm{C}$ for 1 minute, $72^{\circ} \mathrm{C}$ for 1 minute, and extension at $72^{\circ} \mathrm{C}$ for 10 minutes for a total of 35 cycles. PCR products were digested using 0.5 EcoRV endonuclease enzyme (New England Biolabs) at $37^{\circ} \mathrm{C}$ for 3 hours and digested products were separated on $3.5 \%$ agarose gel stained with ethidium bromide. Wild-type TKD results in 68bp and 49bp product after restriction digestion. In the presence of heterozygous mutation, three bands corresponding to $114 \mathrm{bp}$, 68bp, and 49bp were observed, while in homozygous mutation, FLT3-D835 allele produced only one band corresponding to $114 \mathrm{bp}$.

\section{Statistical Analysis}

Categorical variables were expressed in percentages, median, mean, and standard deviation and compared using student's $t$-test, chi-squared test, and Fisher's exact test. $p$-Value $<0.05$ was considered as statistically significant. Analysis of data was done by SPSS version 21.0. Survival analysis was performed by Kaplan-Meier method.

\section{Results}

A total of 424 de novo patients of AML with an age range of one to 77 years (mean age: 35 years) were tested for FTL3-ITD and FLT3-TKD gene mutations by PCR method. Of 424, 92 (21.7\%) were children and adolescence (19 years and below) and 332 (78.3\%) patients were adults. A male-to-female ratio was 0.9:1 (210 man: 214 woman). Among 424 patients, 97 patients (22.9\%) showed FLT3 (any) mutation, of which 70 (72.2\%) and 25 (25.7\%) patients had FLT3-ITD and FLT3-TKD mutation, respectively. Remaining two (2.1\%) patients showed double mutation having both ITD and TKD.

A significant association was found between FLT3 gene mutation and hepatomegaly $(p<0.001)$ and/or splenomegaly $(p<0.001)$ detected by ultrasonography. Patients with FLT3-ITD mutation showed high tumor burden characterized by higher total leucocyte count $(p<0.001)$, higher blast percentages in peripheral blood $(p=0.01)$ as well as in bone marrow ( $p=0.03$ ), and higher serum LDH levels ( $p=0.10)$ compared with FLT3-negative patients (-Table 1). The former group also showed higher platelet count $(p<0.001)$ compared with FLT3-negative patients. However, patients having FLT3-TKD mutation lacked such differences from FLT3-negative cases, except only in higher blast percentage in peripheral blood $(p=0.02)$ (-Table 2 ).

Table 1 Comparison of hematological parameters and LDH between FLT3-ITD positive and FLT3-negative patients

\begin{tabular}{|c|c|c|c|}
\hline Parameters & $\begin{array}{l}\text { Mean } \\
\text { FLT3-ITD, } n=72(16.9 \%)\end{array}$ & $\begin{array}{l}\text { Mean } \\
\text { FLT3 negative, } n=327 \text { (77.1\%) }\end{array}$ & $p$-Value \\
\hline Hemoglobin $(\mathrm{g} / \mathrm{L})$ & 77 & 76 & $0.00^{\mathrm{a}}$ \\
\hline 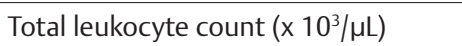 & 81.9 & 46.2 & $0.00^{\mathrm{a}}$ \\
\hline Platelet count $\left(\times 10^{3} / \mu \mathrm{L}\right)$ & 126.8 & 66.9 & $0.00^{\mathrm{a}}$ \\
\hline Blast percentage in blood (\%) & 64.5 & 49.7 & $0.01^{\mathrm{a}}$ \\
\hline Blast percentage in bone marrow (\%) & 70.1 & 56.1 & $0.03^{a}$ \\
\hline $\mathrm{LDH}(\mathrm{U} / \mathrm{L})$ & 778 & 586 & 0.10 \\
\hline
\end{tabular}

Abbreviations: FLT3-ITD, FMS-like tyrosine kinase 3-internal tandem duplication; LDH, lactate dehydrogenase.

${ }^{\text {a }}$-Value $<0.05$ is considered statistically significant.

Table 2 Comparison of hematological parameters and LDH between FLT3-TKD positive and FLT3-negative patients

\begin{tabular}{|c|c|c|c|}
\hline Parameters & $\begin{array}{l}\text { Mean } \\
\text { FLT3-TKD, } n=25 \text { (5.9\%) }\end{array}$ & $\begin{array}{l}\text { Mean } \\
\text { FLT3 negative, } n=327 \text { (77.1\%) }\end{array}$ & $p$-Value \\
\hline Hemoglobin $(\mathrm{g} / \mathrm{L})$ & 79 & 76 & $0.00^{\mathrm{a}}$ \\
\hline 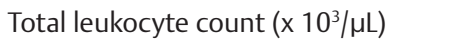 & 63.1 & 46.2 & 0.40 \\
\hline Platelet count $\left(\times 10^{3} / \mu \mathrm{L}\right)$ & 84 & 66.9 & 0.35 \\
\hline Blast percentage in blood (\%) & 60.7 & 49.7 & $0.02^{\mathrm{a}}$ \\
\hline Blast percentage in bone marrow (\%) & 68.1 & 56.1 & 0.18 \\
\hline $\mathrm{LDH}(\mathrm{U} / \mathrm{L})$ & 764 & 586 & 0.37 \\
\hline
\end{tabular}

Abbreviations: FLT3-TKD, FMS-like tyrosine kinase 3-tyrosine kinase domain; LDH, lactate dehydrogenase.

a $p$-Value $<0.05$ is considered statistically significant. 
Morphologically blasts of AML-M4/M5 category of FAB were more common in patients with FLT3 mutation (61/97, 62.9\%) compared with FLT3-negative (80/327, 24.5\%) patients. Immunophenotypically, blasts in FLT3 mutated patients showed consistent expression of monocytic markers (CD 14, CD64) along with strong expression of CD13 and CD33. We also found that higher expression of CD7 $(p=0.06)$, coexpression of CD7 with CD117 $(p=0.01)$, and absent or reduced expression of CD34 ( $p<0.001)$ were associated with FLT3 mutation, suggesting that they can serve as predictive markers for FLT3 mutation in AML (-Fig. 1). Immunophenotypic expression of markers in FLT3 mutation is summarized in - Table 3.

Cytogenetic analysis was performed on all AML patients and were divided into three cytogenetic subgroups. Among FLT3-positive patients, 63.9\% (62/97) had NK, 9.3\% (9/97) patients harbored rearrangement of CBF having either $\mathrm{t}(8 ; 21)$ or $\operatorname{Inv}(16)$ or $\mathrm{t}(16 ; 16)$, and $2.0 \%$ (2/97) of patients belonged to high-risk group. Remaining 24.7\% (24/97) patients had other chromosomal abnormalities. In the favorable CBF rearranged subgroup, seven out of nine patients harbored FLT3-TKD mutation.

Survival analysis of AML patients was performed on 424 AML patients by Kaplan-Meier method. In the current study, OS of all AML patients was 67\%. OS of FLT3-negative patients and FLT3-TKD patients were 69.8 and 64\%, respectively. In contrast, patients harboring FLT3-ITD mutation had worse outcome with OS of 55.6\%. Survival analysis was also done in different cytogenetic subgroups, males versus females and in younger patients ( $<60$ years) versus older
Table 3 Immunophenotypic characteristics of FLT3 patients

\begin{tabular}{|l|l|l|l|}
\hline Markers & $\begin{array}{l}\text { FLT3 positive, } \\
n=97(22.9 \%)\end{array}$ & $\begin{array}{l}\text { FLT3 negative, } \\
n=327(77.1 \%)\end{array}$ & $p$-Value \\
\hline CD 13, $n(\%)$ & $68(70 \%)$ & $244(74.6 \%)$ & 0.09 \\
\hline CD 33, $n(\%)$ & $69(71.1 \%)$ & $244(74.6 \%)$ & 0.18 \\
\hline CD 34, $n(\%)$ & $9(9.3 \%)$ & $185(56.6 \%)$ & $<0.001^{\text {a }}$ \\
\hline HLADR, $n(\%)$ & $61(62.9 \%)$ & $214(65.4 \%)$ & 0.38 \\
\hline CD 117, $n(\%)$ & $63(64.9 \%)$ & $227(69.4 \%)$ & 0.12 \\
\hline CD 7, $n(\%)$ & $21(21.6 \%)$ & $57(17.4 \%)$ & 0.06 \\
\hline $\begin{array}{l}\text { CD7 + CD117, } \\
n(\%)\end{array}$ & $21(21.6 \%)$ & $52(15.9 \%)$ & $0.01^{\text {a }}$ \\
\hline CD 19, $n(\%)$ & $5(5.2 \%)$ & $14(4.3 \%)$ & 0.49 \\
\hline
\end{tabular}

Abbreviation: FLT3, FMS-like tyrosine kinase 3.

${ }^{a} p$-Value $<0.05$ is considered statistically significant.

patients ( $>60$ years). The findings of survival analysis have been depicted in -Fig. 2.

\section{Discussion}

Mutation in FMS-like tyrosine kinase-3 genes is one of the most frequently encountered and clinically challenging mutations in AML. There are two categories of FLT3 mutations, FLT3-internal tandem duplication (FLT3-ITD), and point mutation FLT3-TKD. While FLT3-ITD mutation of juxtamembrane segment is one of the most common mutations found in AML patients, with a frequency of $\sim 20$ to $30 \%$, FLT3-TKD mutation occurs in 5 to $10 \%$ of patients. While role of FLT3-ITD in AML is well known, role of TKD is still a controversy. , $^{3,4,9-11}$

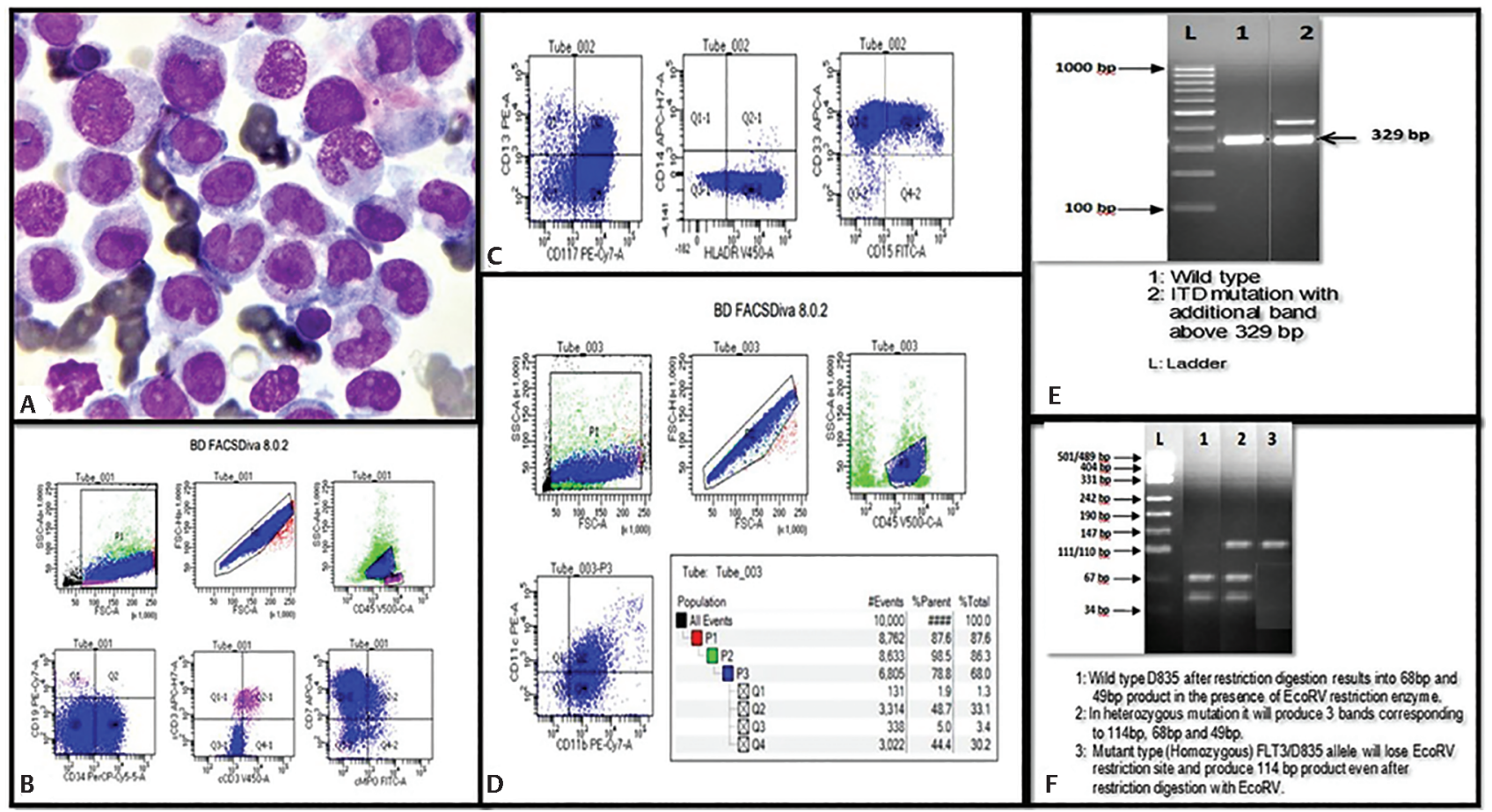

Fig. 1 (A) Bone marrow smear of AML-M5a in a patient with FLT3 mutation (Giemsa stain, 100x). (B-D) Flow cytometric immunophenotyping showing expression of monocytic markers (CD11b, CD11c, an CD15) along with other myeloid markers and aberrant expression of CD7. Gel electrophoresis showing FLT3-ITD mutation (E) and FLT3-TKD mutation (F). bp, base pair; CD, cluster of differentiation; FLT3-, FMS like tyrosine kinase 3; FSC, forward scatter; ITD, internal tandem duplication; SSC, side scatter; TKD, tyrosine kinase domain. 


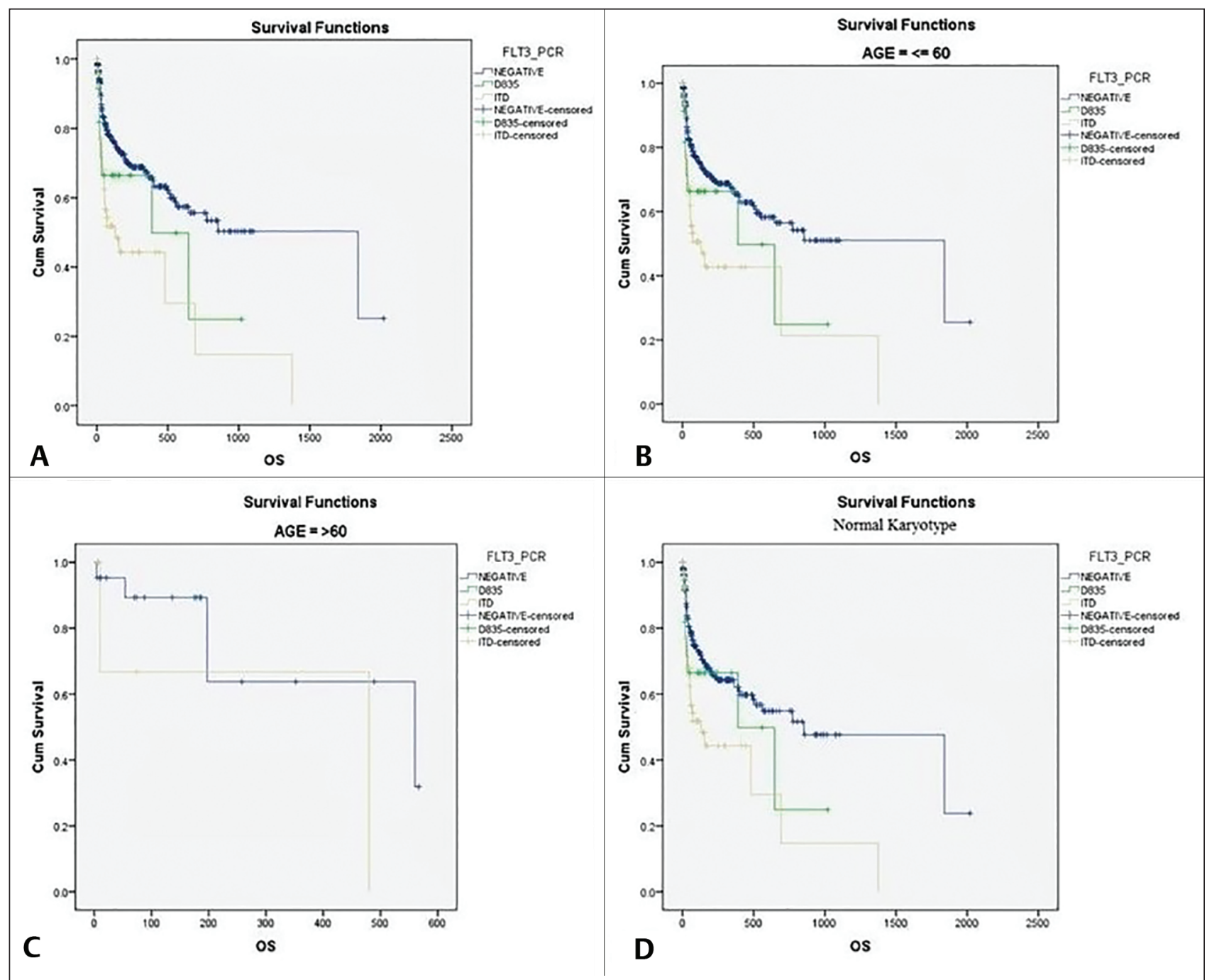

Fig. 2 Survival analysis (Kaplan-Meier method). (A) OS in different groups of AML patients. (B) Survival in younger patients ( $\leq 60$ years) in different groups. (C) Survival in older patients (>60 years). (D) Survival in normal karyotype patients of different groups of AML. AML, acute myeloid leukemia; FLT3, FMS-like tyrosine kinase 3; ITD, internal tandem duplication; PCR, polymerase chain reaction; OS, overall survival.

We analyzed peripheral blood samples of 424 newly diagnosed cases of AML for FLT3-ITD and FLT3-TKD mutations by PCR and PCR-RFLP methods, respectively. The frequency of FLT3 gene mutation in our study was 22.9\% (97/424), similar (23\%) to that documented by Santos et al in a study conducted in the University of Texas in Western population. ${ }^{12}$ However, the frequency documented in our population was higher than that of Assam (16.4\%), a North Eastern state of India as documented in a study conducted by Bhattacharyya et al. ${ }^{8}$ Frequency of 16.5 (70/97) and 5.9\% (25/97) in FLT3-ITD and FLT3-TKD, respectively, was seen in the current study. Two patients $(0.5 \%)$ had dual mutation and both were females. Nakao et al and Meshinchi et al had reported the frequency of 17 and $16.5 \%$, respectively, in FLT3-ITD similar to the current study. ${ }^{13,14}$ Slightly higher frequency was seen in South Indian population (25\%), Japanese (22.6\%), and in Germans (21\%) compared with our study. ${ }^{1,15,16}$ Frequency of 14.6 and $1.8 \%$ in FLT3-ITD and FLT3-TKD, respectively, was seen in people of Assam, which is lower compared with our population. ${ }^{8}$
FLT3-ITD mutation is common in younger age group ( $<60$ years) and serves as an indicator of poor prognosis. Although AML shows male predominance, FLT3-ITD mutation is more common in females. ${ }^{2}$ The mean age at diagnosis in our study was 35 years similar to previous studies. ${ }^{1,8}$ Slight female predominance was seen ( 53.6 vs. $46.4 \%$ ) with a male to female ratio of $0.8: 1$. Younger patients ( $<60$ years) had worse OS compared with older patients ( 54.5 vs. $66.7 \%$ ). This finding is similar to a recently published (2020), population-based cohort study done on Sweden population by Juliusson et al, on 2665 AML patients. They concluded FLT3-ITD mutation to be a poor prognostic marker in younger patients and in females. ${ }^{2}$ However, no significant difference was noted in OS between males and females in the present study (56.4 vs. $54.5 \%$ ).

FLT3 mutation is associated with high tumor burden in AML. Patients with FLT3-ITD mutation had higher white blood counts $(p<0.001)$, higher blast percentage in peripheral blood $(p=0.01)$, as well as bone marrow $(p=0.03)$ 
compared with those without FLT3-ITD mutation. Their serum LDH levels were also higher compared with those without mutation (mean: $777.9 \mathrm{U} / \mathrm{L}$ vs. $586.2 \mathrm{U} / \mathrm{L}, p=0.10$ ). These findings are similar to findings of study conducted by Khera et al and Juliusson et al. ${ }^{1,2}$ FLT3-ITD has an important role in pathogenesis of AML. High tumor burden in FLT3-ITD mutation can be attributed to ligand independent constitutive activation of FLT3 kinase induced by either length mutation in juxta-membrane domain or activation loop mutation of FLT3-ITD. This activates downstream signal molecules that provide survival advantage and result in myeloproliferative phenotype. ${ }^{3,4,17,18}$ In our study, we also found higher platelet count in mutation-positive patients compared with mutation-negative patients (mean $126.8 \times 10^{3} / \mu \mathrm{L}$ vs. $66.9 \times 10^{3} / \mu L, p<0.001$ ). But patients with FLT3-TKD mutation did not show statistically significant difference in blood counts and serum LDH, except for the higher blast percentage in peripheral blood $(p=0.02)$ compared with those without FLT3 mutation. The pathogenetic role of TKD mutation is not well understood and further studies need to be conducted.

Morphologic classification of AML was based on FAB subtypes and standard cytochemical criteria. Myelomonocytic morphology (M4/M5) was the most common FAB subtype seen in our study with the frequency of $62.9 \%(61 / 97)$ followed by M1 (19.6\%, 19/97) and M2 (15.5\%, 15/97). M0 morphology was seen in $2(2.1 \%)$ patients, who were confirmed by immunophenotyping. Sudan Black B was consistently positive in most of the patients. These findings were similar to other studies. ${ }^{19-21}$

Immunophenotypic analysis was done in all 424 patients. The summary of immunophenotyping findings in FLT3 patients is shown in - Table 3. A consistent myelomonocytic phenotype was seen in these patients with frequent expression of CD 64 and CD14. The frequently seen pattern of antigen expression was CD13+ CD33+ CD34- HLADR+ CD117+ CD15+ CD14+ CD64+. The most frequent aberrantly expressed antigen was CD7 (21/97, 21.6\%). All of these patients with aberrant CD 7 also showed coexpression of CD117. Other aberrantly expressed antigens were $\mathrm{CD} 19$ (5/97, 5.2\%) and one each of CD3, CD2, and CD5. Expression of CD34 was variable (9/97, 9.3\%) and weak. These findings were similar to findings observed by Muñoz et al. ${ }^{1,7}$ Hence, CD7 expression with coexpression of CD117 and weak or absent CD34 in the presence of strong CD13 and CD33 act as surrogate markers and predict FLT3 gene mutation in AML.

AML is characterized by clonal evolution and genetic heterogeneity. Genetic abnormalities in AML include amplification, deletions, rearrangements, and point mutations. Prognostic risk at diagnosis is defined by certain recurrent cytogenetic alterations and molecular abnormalities. FLT3-ITD mutation is a poor prognostic molecular genetic alteration significantly affecting patient outcome in AML especially in those harboring normal cytogenetics., ${ }^{3,4,6,12,22}$
Findings of the current study add to the existing data of FLT3 AML. To substantiate the existing notion, we found that OS of patients with FLT3-ITD mutation was much lower compared with FLT3-negative patients (55.6 vs. 69.8). However, , OS of patients with FLT3-TKD mutation was comparable with that of FLT3-negative patients (64 vs. 69.8\%). Survival analysis of AML patients is depicted in - Fig. 2.

The NCCN (National Comprehensive Cancer Network) clinical practice guidelines in oncology classify AML patients bearing FLT3-ITD mutation with NK as poor risk. ${ }^{3}$ We divided 97 FLT3 mutation patients into three cytogenetic subgroups: 62 (63.9\%) cases in NK, 9 (9.3\%) in CBF, and 2 (2\%) in high-risk groups. Remaining 24 (24.7\%) had other cytogenetic abnormalities. OS of NK AML patients who harbored FLT3-ITD mutation was 55.6\% compared with OS (67.5\%) of NK patients who did not show FLT3 mutation as in previous studies. However, FLT3 patients in favorable CBF subgroup did not show significant difference in OS compared with FLT3-negative patients. We found 77.8\% (7/9) of patients in CBF subgroup harbored FLT3-TKD mutation. However, only $24.2 \%$ (15/62) of patients in NK subgroup had FLT3-TKD mutation. This could be one of the reasons that patients with FLT3-TKD mutation do not behave as badly as compared with FLT3-ITD mutation patients. Further studies need to be performed to understand the pathogenesis and influence of other genetic abnormalities in these patients. It is also well known that the pathogenesis and outcome in FLT3-ITD mutation depend on accompanying genetic abnormalities and mutation burden. Analysis of mutation burden helps in further risk categorization of these patients. ${ }^{3,6}$

\section{Conclusion}

FLT3-ITD mutation in AML is associated with high tumor burden and poorer outcomes. Testing for FLT3-ITD mutation at the time of diagnosis has become the standard of care in the management of AML patients in many institutions. But in research-poor settings, presence of certain hematological and immunophenotypic characteristics acts as surrogate markers and can help predict the presence of this mutation in AML patients. Findings of our study add to the existing data of FLT3 mutation in AML.

\section{Funding}

This work was supported by the Department of Immunohematology and Radiodiagnosis, Gujarat Cancer Research Institute, Ahmedabad, Gujarat, India.

\section{Conflict of Interest}

None declared.

\section{Acknowledgment}

The authors acknowledge Mr. Ashish Batham for his contribution in statistical analysis of data. 


\section{References}

1 Khera R, Ahmed F, Mundada M, et al. Acute myeloid leukaemia with gene mutation: a correlation with haematological and immunophenotypic characteristics and our experience in a tertiary care cancer center in South India. Turk Patoloji Derg 2018;34(2):171-174

2 Juliusson G, Jädersten $M$, Deneberg $S$, et al. The prognostic impact of FLT3-ITD and NPM1 mutation in adult AML is age-dependent in the population-based setting. Blood Adv 2020;4(6):1094-1101

3 Daver N, Schlenk RF, Russell NH, Levis MJ. Targeting FLT3 mutations in AML: review of current knowledge and evidence. Leukemia 2019;33(2):299-312

4 Gilliland DG, Griffin JD. The roles of FLT3 in hematopoiesis and leukemia. Blood 2002;100(5):1532-1542

5 Wu M, Li C, Zhu X. FLT3 inhibitors in acute myeloid leukemia. J Hematol Oncol 2018;11(1):133

6 Liu SB, Dong HJ, Bao XB, et al. Impact of FLT3-ITD length on prognosis of acute myeloid leukemia. Haematologica 2019;104(1):e9-e12

7 Muñoz L, Aventín A, Villamor N, et al. Immunophenotypic findings in acute myeloid leukemia with FLT3 internal tandem duplication. Haematologica 2003;88(6):637-645

8 Bhattacharyya J, Nath S, Saikia KK, et al. Prevalence and clinical significance of FLT3 and NPM1 mutations in acute myeloid leukaemia patients of Assam, India. Indian J Hematol Blood Transfus 2018;34(1):32-42

9 Dash A, Gilliland DG. Molecular genetics of acute myeloid leukaemia. Best Pract Res Clin Haematol 2001;14(1):49-64

10 Hayakawa F, Towatari M, Kiyoi H, et al. Tandem-duplicated Flt3 constitutively activates STAT5 and MAP kinase and introduces autonomous cell growth in IL-3-dependent cell lines. Oncogene 2000;19(5):624-631

11 Turner AM, Lin NL, Issarachai S, Lyman SD, Broudy VC. FLT3 receptor expression on the surface of normal and malignant human hematopoietic cells. Blood 1996;88(9):3383-3390
12 Santos FPS, Jones D, Qiao W, et al. Prognostic value of FLT3 mutations among different cytogenetic subgroups in acute myeloid leukemia. Cancer 2011;117(10):2145-2155

13 Nakao M, Yokota S, Iwai T, et al. Internal tandem duplication of the flt3 gene found in acute myeloid leukemia. Leukemia 1996;10(12):1911-1918

14 Meshinchi S, Woods WG, Stirewalt DL, et al. Prevalence and prognostic significance of Flt3 internal tandem duplication in pediatric acute myeloid leukemia. Blood 2001;97(1):89-94

15 Suzuki T, Kiyoi H, Ozeki K, et al. Clinical characteristics and prognostic implications of NPM1 mutations in acute myeloid leukemia. Blood 2005;106(8):2854-2861

16 Thiede C, Koch S, Creutzig E, et al. Prevalence and prognostic impact of NPM1 mutations in 1485 adult patients with acute myeloid leukemia (AML) Blood 2006;107(10):4011-4020

17 Drexler HG. Expression of FLT3 receptor and response to FLT3 ligand by leukemic cells. Leukemia 1996;10(4):588-599

18 Kiyoi H, Towatari M, Yokota S, et al. Internal tandem duplication of the FLT3 gene is a novel modality of elongation mutation which causes constitutive activation of the product. Leukemia 1998;12(9):1333-1337

19 Kuchenbauer F, Kern W, Schoch C, et al. Detailed analysis of FLT3 expression levels in acute myeloid leukemia. Haematologica 2005;90(12):1617-1625

20 Zwaan CM, Meshinchi S, Radich JP, et al. FLT3 internal tandem duplication in 234 children with acute myeloid leukemia: prognostic significance and relation to cellular drug resistance. Blood 2003;102(7):2387-2394

21 Schnittger S, Schoch C, Dugas M, et al. Analysis of FLT3 length mutations in 1003 patients with acute myeloid leukemia: correlation to cytogenetics, FAB subtype, and prognosis in the AMLCG study and usefulness as a marker for the detection of minimal residual disease. Blood 2002;100(1):59-66

22 Warren M, Luthra R, Yin CC, et al. Clinical impact of change of FLT3 mutation status in acute myeloid leukemia patients. Mod Pathol 2012;25(10):1405-1412 